Data de recebimento do artigo: $26-09-2018$

Data de aceite do artigo: 20-02-2019

\title{
Orientação e gestão para stakeholders no processo de decisão organizacional
}

\author{
Fabricio Stocker \\ Doutorando em Administração na Faculdade de Economia, Administração e Contabilidade da \\ Universidade de São Paulo (FEA/USP). E-mail: fabriciostocker@ hotmail.com (Brasil)

\section{Keysa Manuela Cunha de Mascena} \\ Doutora em Administração pela Faculdade de Economia, Administração e Contabilidade da \\ Universidade de São Paulo (FEA/USP). Professora do Programa de Pós-Graduação em \\ Administração de Empresas da Universidade de Fortaleza (Unifor). \\ E-mail: keysamascena@unifor.br (Brasil)
}

\section{Resumo}

A participação dos stakeholders no processo de decisão tem sido recomendada por trazer vantagens competitivas para as organizações. Nesse sentido, esta pesquisa tem por objetivo entender qual a relação entre a gestão para stakeholders e a participação no processo de decisão. Para tanto, foi desenvolvido um ensaio teórico com o objetivo de levantar proposições de pesquisa e reflexões teóricas sobre o tema. Com este trabalho busca-se instigar pesquisas sobre as seguintes proposições: Proposição 1: Quanto maior a prioridade do stakeholder maior a participação no processo de decisão organizacional; Proposição 2: Quanto maior a reciprocidade no relacionamento com o stakeholder maior a participação no processo de decisão organizacional; Proposição 3: O tipo de stakeholder modera a relação entre a prioridade do stakeholder e a participação no processo de decisão organizacional e Proposição 4: As características do stakeholder moderam a relação entre a reciprocidade no relacionamento com o stakeholder e a participação no processo de decisão organizacional. Embora o trabalho discuta o tema ainda de forma embrionária, pode-se verificar um potencial de investigação para as questões que o permeiam. Uma dessas questões refere-se aos limites para a participação dos stakeholders na tomada de decisão para que haja benefício mútuo em vez do aumento de conflitos de interesses ou de alianças políticas. Outro ponto é como operacionalizar empiricamente e operar gerencialmente um relacionamento de reciprocidade entre a organização e seus stakeholders.

Palavras-chave: Gestão para stakeholders. Teoria de stakeholders. Tomada de decisão. 


\title{
Orientation and managing for stakeholders in the decision-making process
}

\begin{abstract}
The stakeholders' participation in decision process has been recommended to bring competitive advantages to organizations. In this sense, is the aim of this paper is to understand the relationship between management for stakeholders and participation in decision process. To address this issue, this article develops a theoretical essay with the objective of raising research propositions and theoretical reflections on the subject. This research seeks to develop and instigate research on the following propositions: Proposition 1: The higher priority of the stakeholder the greater the participation in decision process; Proposition 2: The greater reciprocity in the relationship with the stakeholder the greater the participation in the decision process; Proposition 3: The type of stakeholder moderates the relationship between stakeholder priority and participation in decision process. Proposition 4: The characteristics of stakeholder reinforce the relationship between reciprocity in the stakeholder relationship and participation in decision process. Although the paper discusses the topic still in an embryonic way, one can verify a potential investigation of issues that permeate the subject. One of these questions refers to the limits on stakeholder participation in decision-making so that there is mutual benefit rather than increased conflicts of interest or political alliances. Another point is how to empirically operate and operate a reciprocal relationship between the organization and the stakeholders.
\end{abstract}

Keywords: Decision making. Managing for stakeholders. Stakeholder Theory.

\section{Como referenciar em APA:}

Stocker, F., \& Mascena, K. M. C. de (2019). Orientação e gestão para stakeholders no processo de decisão organizacional. R.G.Secr.,GESEC, 10(1), 167-191.

\section{Como referenciar em ABNT:}

STOCKER, F.; MASCENA, K. M. C. Orientação e gestão para stakeholders no processo de decisão organizacional. R.G.Secr.,GESEC, São Paulo, v. 10, n. 1, p. 167-191, jan.-abr. 2019.
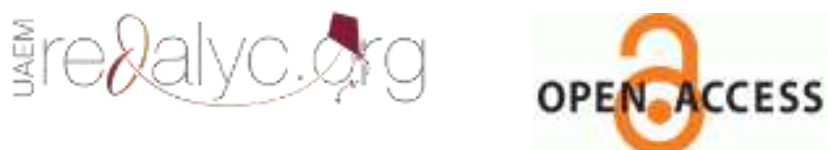


\section{Introdução}

A literatura da teoria dos stakeholders, a partir da década de 1980, vem destacando importância para a maneira com que as empresas se relacionam com seus stakeholders, e como a geração e distribuição de valor é realizada para além da perspectiva dos shareholders (Parmar, Freeman, Harrison, Wicks, Purnell, \& De Colle, 2010). Esse conflito refletia-se na oposição entre a proposição da shareholder view, de que o objetivo da firma deveria ser a maximização da riqueza dos acionistas, e a proposição da stakeholder view, de que a firma deveria ser um veículo de coordenação dos interesses dos stakeholders (Beaucamp \& Bowie, 2001; Windson, 2017). Um dos críticos da stakeholder view, Jensen (2001) alega que o objetivo de coordenação dos interesses dos stakeholders não deixava claro para os gestores quais os critérios para tomada de decisão. Sundaram e Inkpen (2004) acrescentam que a função-objetivo de maximização de valor dos acionistas oferece metas e critérios de decisão para os gestores na formulação e implementação da estratégia corporativa e incentiva os gestores a assumirem riscos.

Por outro lado, na literatura recente, autores como Freeman (2010), Jones, Wicks e Freeman (2017) Jones, Harrison \& Felps (2018) defendem que na gestão para stakeholders deve-se pensar que não há trade-offs. Para Freeman (2010, p. 8) na visão dos economistas sempre haverá trade-offs pois, por exemplo, ao optar por produzir um produto consequentemente deixa-se de produzir outro. No entanto, segundo o autor, os gestores enfrentam múltiplas demandas simultaneamente e devem buscar descobrir como atender às demandas dos clientes, fornecedores, funcionários, comunidades e financiadores, de modo que todos ganhem. Diante de situações complexas, Freeman (2010) ressalta que mesmo os stakeholders mais difíceis de agradar ou com valores conflitantes podem ser fontes de criação de valor, se abordados segundo o mindset da gestão para stakeholders de "no trade-offs", ou seja, que não tem o foco em trade-offs mas na criação de valor para stakeholders.

Nesse modelo de gestão, teóricos como Phillips, Freeman, \& Wicks (2003), argumentam que as premissas defendidas pela teoria de stakeholders vão no sentido de aumentar valor para cada stakeholder sem que haja diminuição para o outro, ou seja, o modelo de gestão da organização deve buscar alternativas para atender os interesses de mais de um stakeholder simultaneamente, possibilitando maior criação de valor.

Corroborando com a proposição de Freeman (2010), Tantalo e Priem (2016) alegam que o gap desta abordagem está no "how", ou seja, como trabalhar na prática essa abordagem de 
criação de valor. Os autores propõem que este gap pode ser preenchido pelo conhecimento das funções utilidades dos stakeholders. Considerando-se que os stakeholders têm funções utilidade multi-atributo, há oportunidades de criar valor para dois ou mais grupos de stakeholders simultaneamente, e sem trade-offs, obtendo-se a sinergia de stakeholders (Tantalo \& Priem, 2016).

Pesquisadores como Bridoux e Stoelhorst (2016) e Rühli, Sachs, Schmitt \& Schneider. (2017) buscam entender como o tipo de relacionamento e nível de confiança geram diferentes impactos no relacionamento entre os stakeholders e os gestores das organizações. Ainda assim, permanece válida a premissa apresentada por Harrison, Bosse e Phillips (2010), que para que os stakeholders compartilhem informações sobre sua função utilidade, a empresa deve manter um relacionamento com os stakeholders baseado em confiança e reciprocidade (também no sentido de justiça - fairness), e este compartilhamento de informações dos stakeholders levaria a uma alocação mais eficiente de recursos e a obtenção de vantagens competitivas.

Nesse processo de gestão e alocação de recursos de forma distributiva, o envolvimento e participação dos stakeholders se mostra primordial, não só pelo compartilhamento de informações como também pelo engajamento na tomada de decisão e nos objetivos estratégicos da organização (Ashmos, Duchon, \& McDaniel, 1998). MacDonald, Clarke e Huang (2018) destacam que o envolvimento e participação de stakeholders na tomada de decisão têm sido recomendada para trazer vantagens competitivas às organizações, além dos ganhos processuais pelo envolvimento dos stakeholders e compartilhamento de informações.

Frente a estes argumentos, esse estudo se posiciona na fronteira da discussão do campo teórico da teoria de stakeholders e busca entender como a gestão para stakeholders leva à inclusão destes na participação na tomada de decisão da organização e como as nuances do relacionamento e da priorização de stakeholders pode ter influência no compartilhamento de informações e no engajamento destes no processo de decisão organizacional. Para abordar esta problemática, nesse artigo é desenvolvido um ensaio teórico com o objetivo de levantar proposições de pesquisa e reflexões teóricas sobre o tema.

\section{Fundamentação Teórica}

O referencial teórico desta pesquisa abrange a discussão tradicional e ainda não encerrada, sobre as visões de stakeholders e shareholders quanto ao propósito da organização, busca-se ainda apresentar e discutir como a teoria dos stakeholders sugere como deve ser a 
priorização dos stakeholders, de forma a lidar melhor com os conflitos das relações entre estes e a organização. A virada de trade-offs para o mindset da gestão para stakeholders e como essa abordagem teórica tem como finalidade garantir a vantagem competitiva do negócio. Por fim é apresentado também o tópico sobre o engajamento com stakeholders e o envolvimento destes no processo de decisão organizacional.

\subsection{Visão de stakeholders versus visão de shareholders}

Em sua obra, Freeman (1984) discute a questão da primazia do shareholder, owners ou acionistas, ao questionar se gestores deveriam atentar-se apenas para os interesses dos acionistas ou deveriam considerar os interesses dos demais stakeholders.

Esta discussão é desenvolvida, em obra posterior de Evan e Freeman (1993) que propõem que a função-objetivo da firma deveria ser a de servir como um veículo para coordenação dos interesses dos stakeholders. Ao empregar o termo coordenação, os autores não deixam claros critérios de priorização, mas rejeitam a primazia do acionista como proposto pela função-objetivo da teoria da firma.

Jensen (2001) afirma que a empresa deve ter foco em apenas um objetivo, e não em múltiplos objetivos, e esse objetivo único seria a maximização do valor da empresa, que por sua vez maximizaria o bem-estar social. Para o autor, ter múltiplos objetivos, conforme alega ser sugerido pela teoria dos stakeholders, significa não ter objetivos.

Sundaram e Inkpen (2004) acrescentam que a função-objetivo de maximização de valor dos acionistas oferece metas e critérios de decisão para os gestores na formulação e implementação da estratégia corporativa e incentiva os gestores a assumirem riscos.

Em contrapartida, na perspectiva dos stakeholders, a visão associada à teoria da firma falha ao enfatizar apenas um stakeholder: o acionista. Outras críticas também apontam que os gestores têm dever fiduciário com a corporação e não com os acionistas (Beaucamp \& Bowie, 2001).

Embora haja contraposição entre as duas teorias, Jensen (2001) propõe uma abordagem de convergência. $\mathrm{O}$ autor reconhece que a empresa não alcançará a maximização do seu valor se não levar em conta os interesses dos stakeholders, e então sugere a enlightened stakeholder theory. Segundo essa proposta, a empresa deverá preocupar-se com o atendimento dos interesses dos stakeholders, porém, sua função-objetivo ainda seria a maximização do valor de 
longo prazo da empresa, sendo também o critério adotado para tomada de decisão em casos de interesses conflitantes.

O embate entre a visão de stakeholders e a visão de shareholders é criticado por Freeman (2010), que considera que a principal contribuição da teoria dos stakeholders não é propor diferentes teorias da firma, e sim a combinação dos interesses dos stakeholders. Para o autor, negócios referem-se a como os stakeholders e gestores interagem e criam valor.

O argumento de Freeman (2010) é que, vendo a organização como um nexo de contratos, surge a indagação do que seria um contrato justo. Desta forma, a ética empresarial passou a rejeitar a visão de shareholders e adotar a visão dos stakeholders como um meio de trazer ética e justiça aos negócios.

Na intenção de posicionar e esclarecer o que é a Teoria de Stakeholders e principalmente o que não é, ou seja, do que não se trata a teoria, Phillips, Freeman e Wicks (2003) reforçam que a firma, deve ser vista como um nexo de relacionamentos entre os stakeholders primários com o objetivo de criação de valor. Parmar et al. (2010), contribuem com um importante posicionamento sobre o estado da arte da teoria, afirmando que a teoria de stakeholders é uma narrativa alternativa para entender e buscar melhorar alguns conflitos nos negócios, como por exemplo como o valor é criado e distribuído, como há conexão entre o discurso ético e os preceitos capitalistas e como os gestores e os stakeholders podem lidar para resolver estes dilemas presentes no ambiente das organizações.

\subsection{A orientação na gestão para stakeholders}

A gestão para stakeholders (managing for stakeholders) termo usado por Freeman, Harrison e Wicks (2007) para diferenciar do termo gestão de stakeholders (stakeholder management), que é mais usado na literatura, segundo os autores, envolve a alocação de valor e de influência na tomada de decisão para os stakeholders primários. Nesse sentido, a gestão para stakeholders é a forma de relacionamento pela qual os autores citam ao tratar de não tradeoffs, ou seja, não há conflito entre o objetivo e propósito da firma, seja na visão do shareholder como dos próprios stakeholders.

Harrison, Bosse e Phillips (2010) fazem uma discussão teórica sobre qual tipo de tratamento dado aos stakeholders levaria a obtenção de vantagens competitivas, baseando-se no conceito de gestão para stakeholders de Freeman et al. (2007). O tratamento dado aos stakeholders defendido por Harrison, Bosse e Phillips (2010) é baseado na reciprocidade e na 
justiça distributiva, que refere-se à alocação de valor aos stakeholders fundamentado em sua contribuição para a criação de valor da organização focal.

A contribuição destes autores, ainda se dá no sentido de complementar o conceito de managing for stakeholders, ao declarar que "as empresas que fazem gestão para stakeholders, alocam mais valor do que o necessário, simplesmente para manter sua participação voluntária no funcionamento da empresa" (Harrison, Bosse, \& Phillips, 2010, p. 61).

Segundo os autores, a abordagem de gestão para stakeholders está em contraste com o que pode ser chamado de uma abordagem arms-length, que parte do ponto de vista, em que os stakeholders são considerados atores econômicos intercambiáveis. Nessa visão de arms-length, o poder é o principal critério em relação à influência sobre as decisões e distribuição de valor da organização. Dessa forma, os stakeholders são envolvidos quando apenas for de interesse da empresa e o valor distribuído é levado em conta sobre os próprios interesses nas negociações.

Em contrapartida, Harrison, Bosse e Phillips (2010), argumentam que por meio de um relacionamento alicerçado em reciprocidade e justiça com os stakeholders primários, a organização tem mais acesso à informações sobre a função utilidade dos stakeholders, gerando potenciais vantagens como o aumento de demanda e eficiência, a inovação e a habilidade de lidar com situações inesperadas. As vantagens obtidas, quando aproveitadas pela organização, geram valor que é distribuído de forma justa entre os todos os stakeholders (Kleine, Langenbach, \& Zhurakhovska, 2016).

Outra consideração é de que, embora ao manter um relacionamento justo com os stakeholders a empresa seja recompensada positivamente, aumentando sua renda total, estes processos justos adicionam custos (Bosse, Phillips, \& Harrison, 2009). Esses custos são decorrentes do aumento do tempo e atenção que os gestores despendem para os stakeholders, do aumento dos custos de comunicação e de processamento de informação, assim como do montante adicional de tempo que leva para a empresa tomar decisões quanto mais contribuições aos stakeholders são consideradas (Harrison \& Bosse, 2013).

Por fim, pode-se dizer que a orientação na gestão para stakeholders se dá no alcance do equilíbrio, justiça e harmonia com todos os mecanismos de relacionamento com os stakeholders (Freeman, Phillips \& Sisodia, 2018). Assim, interessa saber a forma como os gestores percebem de forma diferente quais stakeholders são mais salientes no processo de priorização, como se dá o engajamento com estes atores e de que forma e em qual profundidade eles conseguem 
envolver os stakeholders nos processos de decisão organizacional. Fatores estes que serão especificados nos tópicos a seguir.

\subsection{Priorização de stakeholders}

O conceito de gestão para stakeholders, proposto na obra de Freeman (1984) e revisitado por Freeman, Wicks, \& Parmar (2004) e Parmar et al. (2010), trata-se do relacionamento da empresa com seus stakeholders. Para estabelecer este relacionamento, Freeman (1984) propõe um processo estratégico que inclui identificação e análise de stakeholders, desenvolvimento de estratégias e planos de ação e implementação e monitoramento das estratégias. Ao tratar da implementação estratégica, entende-se que a questão da priorização é discutida pelo autor, ainda que de forma implícita, ao afirmar que os recursos devem ser direcionados aos stakeholders mais importantes, ou seja, aqueles que são vitais para o sucesso futuro da empresa (Freeman, 1984, p. 159).

Segundo Phillips (2003a), uma questão que historicamente desperta atenção na teoria refere-se a como os gestores alocam tempo, atenção, capacidade e outros recursos escassos entre os stakeholders. Segundo o autor os gestores podem priorizar os interesses de alguns stakeholders, seguindo o de tratamento meritocrático e distribuição justa.

Neste sentido, um dos modelos de priorização mais citados na literatura é o modelo de Mitchell, Agle e Wood (1997, p. 854), denominado de saliência dos stakeholders, e definido como o "grau em que os gestores dão prioridade às reivindicações concorrentes dos stakeholders". Segundo os autores, os stakeholders podem ser identificados a partir de três atributos: (1) o poder dos stakeholders de influenciar a organização; (2) a legitimidade da relação do stakeholder com a organização; e (3) a urgência das reivindicações do stakeholder na organização. Dessa forma, a saliência do stakeholder está positivamente relacionada com a percepção gerencial da presença dos três atributos.

Mitchell et al. (1997) desenvolveram uma classificação a partir desses três atributos. Inicialmente, os stakeholders que possuem apenas um atributo, são chamados de stakeholders latentes; aqueles que possuem dois atributos são os stakeholders expectantes; os que possuem os três atributos são os stakeholders definitivos. Segundo o modelo, os gestores possuem claramente o dever de atender e priorizar os interesses dos stakeholders definitivos, ou seja, que possuem poder, legitimidade e urgência. 
Ao fazer um levantamento da produção sobre priorização de stakeholders, Mascena, Kim, Fischmann e Corrêa (2015) argumentaram que, de acordo com as contribuições de diversos estudos sobre priorização, pode-se afirmar que a percepção dos gestores quanto à importância dos stakeholders é influenciada por características dos stakeholders, como a sua saliência (Mitchell et al., 1997), poder (Frooman, 2010; Chigona, Roode, Nabeel, \& Pinnock, 2010) e a legitimidade de suas demandas (Phillips, 2003b).

A percepção de importância dos gestores também é influenciada pelo comportamento dos stakeholders, como a influência do stakeholder na organização (Chigona et al., 2010; Pedersen, 2011; Saravanamuthu, 2018), a pressão dos stakeholders (Murillo-Luna, GarcesAyerbe, \& Rivera-Torres, 2008; Vazquez-Brust, Liston-Heyes, Plaza-Úbeda, \& BurgosJiménez, 2010) e a contribuição do stakeholder para a empresa (Phillips, 2003b; Cameron, Crawley, Feng, \& Lin, 2011). A priorização dos stakeholders também pode ser afetada por características da empresa como a sua função-objetivo (Boaventura, Cardoso, Silva, \& Silva, 2009) e a sua estratégia em relação ao ambiente (Henriques \& Sadorsky, 1999; Buysse \& Verbeke, 2003; Yu, Guo, \& Luu,2018).

\subsection{Engajamento de stakeholders}

O processo ou estratégia de obter vantagem competitiva através do desenvolvimento de relacionamentos com stakeholders é chamado de engajamento de stakeholders (Heugens, Van Den Bosch, \& Van Riel, 2002). O ímpeto por trás do uso do termo "engajamento" na teoria de stakeholders e na literatura sobre responsabilidade social corporativa (CSR) é a necessidade de enfatizar que, para as empresas simplesmente interagirem com as partes interessadas, não é mais suficiente, se de fato já foi. Assim, como consequência, além de pensar sobre quais ações as empresas devem e não devem realizar para atender aos padrões morais, a atenção agora está sendo dada às relações que as empresas devem promover com seus stakeholders (Noland \& Phillips, 2010).

A abordagem para estudar o engajamento de stakeholders considera que o desenvolvimento de iniciativas é realizado em torno de dois focos: comunicação e relacionamento. Essa perspectiva é usada em vários artigos para caracterizar iniciativas de engajamento de stakeholders (Morsing \& Schultz, 2006; Rasche \& Esser, 2006; Huijstee \& Glasbergen, 2008). Além disso, quatro diferentes abordagens são estabelecidas: comunicação, 
diálogo, consulta e parceria (Greenwood, 2007; Krick, Forstater, Monaghan, \& Sillanpää, 2005). Cada abordagem representa um maior compromisso de ambos os lados para investir em tempo, recursos, aumentar sua exposição ao risco enquanto buscam cooperação entre os relacionamentos.

Greenwood (2007) explica que o engajamento de stakeholders pode ser definido como o conjunto de iniciativas ou práticas que as organizações desenvolvem para envolver seus stakeholders de maneira positiva em suas atividades organizacionais. Friedman e Miles (2006) propõem níveis de engajamento em que se pode inferir que, quanto maior o nível de engajamento, maior a participação na tomada de decisão e nas ações da organização, conforme apresentado na Figura 1.

Figura1 - Escada de engajamento de stakeholders

\begin{tabular}{|c|c|c|}
\hline & Níveis & Descrição do Engajamento \\
\hline \multirow{3}{*}{ 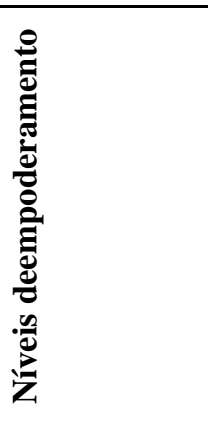 } & $\begin{array}{l}\text { 12. Controle } \\
\text { do stakeholder }\end{array}$ & $\begin{array}{l}\text { Diálogo multi-way. Representação majoritária do stakeholder } \\
\text { no processo de tomada de decisão. P. ex.: projetos } \\
\text { comunitários. }\end{array}$ \\
\hline & $\begin{array}{l}\text { 11. Poder } \\
\text { delegado }\end{array}$ & $\begin{array}{l}\text { Diálogo multi-way. Representação minoritária do stakeholder } \\
\text { no processo de tomada de decisão. P. ex.: representação em } \\
\text { conselho de administração. }\end{array}$ \\
\hline & 10.Parceria & $\begin{array}{l}\text { Diálogo multi-way. Organização e stakeholder tem poder de } \\
\text { decisão conjunta em projetos específicos. P. ex.: joint } \\
\text { ventures. }\end{array}$ \\
\hline \multirow{2}{*}{ 胥 } & $\begin{array}{l}9 . \\
\text { Colaboração }\end{array}$ & $\begin{array}{l}\text { Diálogo multi-way. Algum poder de decisão atribuído ao } \\
\text { stakeholder em projetos específicos. P. ex.: alianças } \\
\text { estratégicas. }\end{array}$ \\
\hline & $\begin{array}{l}8 . \\
\text { Envolvimento }\end{array}$ & $\begin{array}{l}\text { Diálogo multi-way. Apoio condicional do stakeholder, que } \\
\text { pode ser retirado pela organização caso este apoio não se } \\
\text { ajuste aos interesses organizacionais. P. ex.: diálogo } \\
\text { construtivo. }\end{array}$ \\
\hline \multirow{4}{*}{ 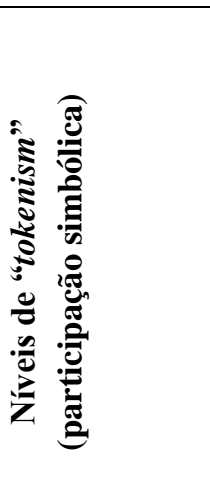 } & 7. Negociação & $\begin{array}{l}\text { Diálogo multi-way.Apoio condicional do stakeholder, que } \\
\text { pode ser retirado pela organização caso este apoio não se } \\
\text { ajuste aos interesses organizacionais. P. ex.: barganha. }\end{array}$ \\
\hline & 6. Consulta & $\begin{array}{l}\text { Diálogo two-way. A organização tem o direito de decidir, os } \\
\text { stakeholders podem apenas opinar. P. ex.: entrevistas, } \\
\text { focusgroups, painéis. }\end{array}$ \\
\hline & 5.Placation & $\begin{array}{l}\text { Diálogo two-way. Stakeholders poder ouvir e serem ouvidos, } \\
\text { mas não há garantias de serem atendidos pela organização. P. } \\
\text { ex.: entrevistas, focusgroups, painéis. }\end{array}$ \\
\hline & 4. Explicação & Diálogotwo-way. "Educar" osstakeholders. P. ex.: workshops. \\
\hline 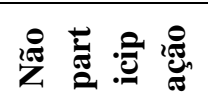 & 3. Informação & $\begin{array}{l}\text { Diálogoone-way. "Educar" osstakeholders. P. ex.: relatórios } \\
\text { de sustentabilidade. }\end{array}$ \\
\hline
\end{tabular}




\begin{tabular}{c|c|l}
\hline \multirow{2}{*}{ 2. Terapia } & $\begin{array}{l}\text { Diálogo one-way. Eliminar dos stakeholders a ignorância e } \\
\text { crenças pré-concebidas da organização. P. ex.: informativos, } \\
\text { reportagens. }\end{array}$ \\
\cline { 2 - 3 } & 1.Manipulação & $\begin{array}{l}\text { Diálogo one-way. Tentativa de modificar as expectativas dos } \\
\text { stakeholders. P. ex.: informativos, reportagens. }\end{array}$ \\
\hline & &
\end{tabular}

Fonte: Friedman \& Miles, 2006, p. 162.

Ao propor os doze níveis de engajamento, Friedman e Miles (2006) ressaltam que a proposta não é que o relacionamento com todos os stakeholders deva subir até o décimo segundo nível, ou até qualquer outro nível “ideal”. Diferentes grupos de stakeholders ou o mesmo grupo em diferentes momentos pode ser gerenciado em diferentes níveis. O que se destaca relevante, nesse sentido, como já defendido por autores como Noland e Phillips (2010), Johnson-Cramer (2004) e Greenwood (2007), é que se faz importante diferenciar a natureza apropriada do engajamento das firmas com seus stakeholders e a qualidade dessas relações, uma vez que essas nuances nas práticas de engajamento, influenciam a maneira como esses stakeholders irão se envolver e estar dispostos a contribuir com os objetivos da organização.

\subsubsection{Participação dos stakeholders na tomada de decisão}

Ao estudar o processo decisório estratégico nas organizações, temos além do campo tradicional sobre o processo da decisão, fatores condicionantes e os aspectos da racionalidade, também fatores pouco discutidos no campo, mas que conforme será abordado a seguir, estão intimamente ligados ao processo decisório.

Para Finkelstein, Hambrick e Cannella (2009), a incerteza característica das situações e decisões complexas inerentes ao processo estratégico dá margem a maiores vieses interpretativos, e um pequeno grupo de pessoas no topo das organizações tende a influenciar fortemente o curso da ação e os resultados organizacionais e, por essa razão, envolver outros atores na tomada de decisão pode ser eficaz na redução dos vieses e também no compartilhamento dos riscos sob as decisões estratégicas.

A participação na tomada de decisão, para Ashmos, Duchon e McDaniel (1998), ocorre de diferentes formas: porcentagem do grupo participante, proporção de tomadores de decisão de cada grupo, amplitude da participação, tempo da participação, atividades (formulação do problema, esclarecimentos, geração de alternativas, avaliação de alternativas, escolha de 
alternativas) e mecanismos (comitês estabelecidos, forças tarefa, reuniões informais, encontros casuais, entre outros).

Considera-se nesse campo de discussão que as decisões são o resultado das ações tomadas pelos gestores - decisores, e estes por sua vez, possuem seus objetivos próprios, podendo ser diferentes dos objetivos da organização e dos stakeholders (Child, Elbanna, \& Rodrigues, 2010). Dessa forma, os objetivos e interesses desses gestores afetam todo o processo decisório nas organizações e envolver outras partes neste processo torna-se importante.

Na visão de Ashmos et al. (1998) o envolvimento com os stakeholders pode ser dar por meio da sua inclusão e participação no processo de tomada de decisão organizacional, o que com base em estudos recentes, como MacDonald, Clarke e Huang (2018) esse envolvimento de stakeholders na tomada de decisões tem sido recomendado para trazer vantagens competitivas para as organizações, seja pelo compartilhamento de informações (Garcia-Castro \& Aguilera, 2015) como pelo envolvimento nos objetivos estratégicos da organização.

A influência do poder e do comportamento político nos processos decisórios dentro da organização, pode ainda levar os gestores a tomarem decisões baseadas em informações incompletas ou até mesmo distorcidas da realidade. Nesse sentido, Abrahamson e Baumard (2008) apresentam como as fachadas organizacionais influenciam as tomadas de decisões e consideram que essas "fachadas" têm como função distorcer fatos e a realidade organizacional aos seus stakeholders.

As fachadas organizacionais representam uma frente simbólica erguida pelos participantes da organização destinadas a tranquilizar os stakeholders da sua organização, da legitimidade e da sua gestão (Nystrom \& Starbuck, 1984). A questão aqui, refere-se ao fato de quando com o uso de fachadas organizacionais, as expectativas do stakeholdersse elevam, e o risco na tomada de decisão organizacional também aumenta (Saravanamuthu, 2018).

Vale ainda ponderar que, embora tradicionalmente o comportamento político e as questões relacionadas ao poder e política, conforme apresentados por Child, Elbanna e Rodrigues (2010) sejam avaliadas em um sentido negativo, é reconhecido que no processo decisório estratégico essas questões estão tão presentes quanto os procedimentos racionais, sendo dessa maneira parte do processo, podendo ser também considerado como fator positivo com relação ao poder de influenciar as decisões estratégicas. O que para a teoria de stakeholders, inclusive é um fator determinando para a priorização dos grupos de stakeholders, conforme já discutido por Mitchell, Agle e Wood (1997). 
Ainda assim, vale ressaltar que o esforço e apelo à participação dos stakeholders nos processos de decisão das empresas podem servir de um propósito duplo: de um lado, a participação permite que as organizações tenham uma melhor compreensão das demandas sociais além das quais ela já espera ter com base naqueles que participam do processo decisório. De outro lado, o envolvimento dos stakeholders na tomada de decisão pode ser utilizado para avaliar o nível de alinhamento entre as demandas e interesses destes, com os objetivos e propósitos da organização em si, situação essa que traduz uma possível vantagem competitiva.

\section{Proposições da Pesquisa}

O envolvimento dos stakeholders na tomada de decisão organizacional pode ser influenciada por diversos fatores. Esta pesquisa buscará investigar as características da orientação na gestão para stakeholders associadas à participação na tomada de decisão. Para tanto, as características da gestão para stakeholders serão consideradas na perspectiva da teoria de stakeholders, sob os aspectos da priorização, engajamento e participação dos stakeholders nas decisões organizacionais.

Conforme defendido por Harrison et al. (2010), as organizações tendem a adotar uma postura de arms-length ou de gestão para stakeholders, sendo que a primeira está mais associada a uma disputa por interesses e a segunda mais associada à uma relação de reciprocidade e justiça. Nesta pesquisa, entende-se que a primeira postura é mais orientada à trade-offs e a segunda menos orientada à trade-offs. Nesse sentido, as proposições são apresentadas, segundo uma gestão orientada à não trade-offs onde a gestão é voltada para beneficiar os interesses de todos stakeholders e que ainda o tipo stakeholder, ainda pode moderar esta relação entre a reciprocidade do relacionamento e sua participação na decisão organizacional.

3.1 Relação entre a priorização do stakeholder e a participação na tomada de decisão

Na gestão para stakeholders, será considerada que a priorização do stakeholder tem relação com a participação na tomada de decisão, pois quanto maior a presença dos atributos que caracterizam a priorização do stakeholder, como importância, poder, e influência, maior seria a probabilidade da organização engajá-lo na tomada de decisão. 
Essa relação está mais alinhada com as organizações orientadas por poder de barganha, ou seja, do tipo arms-length (Bridoux \& Stoelhorst, 2014). Além disso, está ancorada em estudos que tratam da influência dos stakeholders percebidos como prioritários na tomada de decisão (Simons \& Wiegel, 2009; Chakhar \& Saad, 2014; Dye, Eggers \& Shapira, 2014; MacDonald, Clarke \& Huang, 2018).

A relação entre a priorização do stakeholder e a participação na decisão pode ser justificada em função de alguns mecanismos, que são a pressão exercida pelos stakeholders e o poder que eles possuem em relação à empresa.

A pressão dos stakeholders na organização torna as suas demandas mais urgentes e sua participação importante para satisfazer essas demandas. Alguns estudos empíricos abordam a pressão dos stakeholders na tomada de decisão dos gestores, como nos estudos empíricos de Delmas e Toffel (2008) e Stevens, Steensma, Harrison e Cochran (2005). Para Delmas e Toffel (2008), a resposta às demandas do ambiente nas decisões dos facility managers em empresas americanas depende da pressão dos stakeholders. Para Stevens et al. (2005), os executivos de finanças incorporam o código de ética da empresa em seus processos de decisão estratégica se percebem a pressão dos stakeholders para fazê-lo.

O outro mecanismo é o poder do stakeholders, que explica a participação no processo de decisão pelo seu potencial de prejudicar a empresa caso a decisão tomada não corresponda a seus interesses (Cobb, 2016). O estudo empírico de Edelenbos e Klijn (2006) sobre tomada de decisão relacionadas às políticas públicas na Holanda, constatou que um dos motivos para a inclusão dos stakeholders na tomada de decisão é seu poder de veto das decisões tomadas que não os satisfazem.

Uma das fontes de poder do stakeholder é a posse de recursos dos quais a organização depende (Harrison et al., 2010). Os acionistas têm recursos financeiros dos quais a organização depende o que o conduz aos mais altos níveis de engajamento e participação. Um fornecedor poderoso também tem recursos dos quais a empresa depende, o que pode levar a situações de parceria elevando-o a altos níveis de participação na tomada de decisão. Essas características reforçam a percepção de que o stakeholder com poder e que exercem pressão devem ser priorizados. Em função disso, propõe-se que:

Proposição 1: Quanto maior a prioridade do stakeholder maior a participação no processo de decisão organizacional. 
3.2 Relação entre a reciprocidade no relacionamento com o stakeholder e a participação na decisão

$\mathrm{Na}$ gestão orientada a não trade-offs, considera-se que a reciprocidade no relacionamento com o stakeholder tem relação com a participação na decisão, pois quanto maior a relação de parceria e benefício mútuo entre a empresa e o stakeholder, maior seria a probabilidade de a organização engajá-lo na tomada de decisão. Essa relação está mais alinhada com as organizações orientadas por justiça e reciprocidade, ou seja, do tipo fairness (Bridoux \& Stoelhorst, 2014)

Os mecanismos que explicam a relação entre a reciprocidade no relacionamento com o stakeholder e a participação no processo de decisão podem ser justificados em função de alguns mecanismos, que são os valores gerenciais e orientação organizacional. O papel dos valores na tomada de decisão das organizações é objeto de estudo de alguns de estudos empíricos como Adams, Licht e Sagiv (2011), Barbero e Marchiano (2016), O'Brien e David (2014) e Anbarasan (2018).

Adams, Licht e Sagiv (2011) defendem que há dois polos opostos que orientam uma decisão: shareholder view e stakeholder view. A posição dos conselheiros e CEOs no continuum entre estes dois polos opostos são seus valores pessoais. Os valores pessoais são considerados pelos autores de acordo com a classificação de Schwartz (1992). Este argumento é testado em uma amostra de conselheiros e CEOs da Suécia, onde os autores confirmam a hipótese de que a orientação a shareholder está positivamente relacionada com os valores de poder e conquista e negativamente relacionado aos valores de universalismo e benevolência. Portanto, os valores mais associados à stakeholder view são os de universalismo (entendimento, apreciação, tolerância e proteção do bem-estar de todas as pessoas e da natureza) e benevolência (preservação e valorização do bem-estar das pessoas a quem se está em contato pessoal frequente) (Adams et al., 2011, p. 1334).

Sob a mesma lógica, um estudo nacional desenvolvido por Barbero e Marchiano (2016) endereçam a discussão sobre a as características e os valores individuais de conselheiros de empresas de grande porte e de capital aberto do Brasil, e fazem uma possível influencia destas características com a orientação para stakeholders e shareholders. Embora não foi possível concluir a relação entre os valores individuais com a orientação para stakeholders, foi evidenciado que quando os conselheiros têm maior identificação dos valores deles com os 
valores organizacionais, tende a ser mais compromissados com a organização. Nisto reflete-se também a identificação de valores dos demais stakeholders da organização, e com isso, reforçase a premissa sobre a reciprocidade dos relacionamentos na gestão voltada para os stakeholders.

Outro trabalho relacionado com a reciprocidade foi desenvolvido por O’Brien e David (2014) que abordaram os valores culturais das decisões de P\&D no Japão. Para os autores, a reciprocidade e enraizamento podem influenciar a decisão de utilização do desempenho excedente em P\&D. Quando o desempenho excede as aspirações, as empresas orientadas à reciprocidade, como na cultura japonesa, são mais inclinadas a usar a sua posição privilegiada para ajudar stakeholders menos favorecidos. Já no trabalho de Anbarasan (2018) o envolvimento da empresa com os stakeholders, seja no processo de desenvolvimento de ações de engajamento ou simples relacionamento, aponta indícios de uma preocupação com o desenvolvimento sustentável no longo prazo da organização, e que projeto na sua relação com os stakeholders uma melhor forma de prosperar e de criar vantagens competitivas duradouras/sustentáveis.

Além do exposto acima, Harrison e Bosse (2013) reforçam que quando há um relacionamento de reciprocidade com o stakeholder maior o potencial de contribuição do stakeholder para a vantagem competitiva da empresa. Em função disto, oportunidades de parceria e desenvolvimento conjunto podem ser potencializadas levando ao maior engajamento dos stakeholders. A relação de benefício mútuo pode levar a um maior compartilhamento de informações e também a tomada de decisão conjunta, propondo-se desta forma que:

Proposição 2: Quanto maior a reciprocidade no relacionamento com o stakeholder maior a participação no processo de decisão organizacional.

\subsection{As características e o tipo de stakeholder como moderadores da relação}

As relações apresentadas entre priorização e reciprocidade e o processo de decisão podem ter um efeito moderador do tipo e característica dos stakeholders. Esse argumento baseia-se no fato de que as organizações lidam com diferentes stakeholders em diferentes condições de dependência e de relacionamento.

Reforçando o conceito inicial de stakeholders proposto por Freeman (1984, p. 46), temse que "stakeholders são grupos ou indivíduos que podem afetar ou ser afetados pela organização na realização de seus objetivos”. Em complemento a essa definição Friedman e 
Miles (2006, p.10) descrevem com mais precisão a natureza da relação entre a organização e seus stakeholders, referindo-se ao uso do verbo "afetar" no sentido: apoiar, depender, dar significado, beneficiar ou prejudicar, investir ou arriscar algo de valor.

A definição auxilia na identificação dos stakeholders pela organização, sendo que na dimensão instrumental, muitos autores defendem que uma definição mais restrita auxilia os gestores a identificarem os stakeholders principais da empresa. Segundo Clarkson (1995), os stakeholders podem ser classificados como primários ou secundários. Para o autor, os stakeholders primários são aqueles que, sem sua participação, a empresa não poderia sobreviver, havendo alto grau de interdependência entre a corporação e seus stakeholders primários, sendo assim os stakeholders primários: acionistas e investidores, empregados, clientes, fornecedores, comunidades.

Os stakeholders secundários são aqueles que influenciam ou afetam, ou são influenciados ou afetados pela corporação, mas não possuem relação direta com ela. O autor classifica o governo, concorrentes, a mídia, entidades filantrópicas, instituições de ensino e demais grupos de interesse como stakeholders secundários, pois apesar de não serem essenciais para a sobrevivência da organização, podem exercer influência na percepção que os stakeholders primários têm da organização. Freeman (1984) faz a ressalva que as classificações podem ter alguns ajustes em função das características da organização, por exemplo, em determinada organização o governo pode ser primário enquanto em outra o governo é um stakeholder secundário.

Sob outro ponto de vista, Bridoux e Stoelhorst (2014) incluem na pauta de discussão as características individuais dos stakeholders e como isso afeta a maneira como os relacionamentos são desenvolvidos e como os stakeholders se identificam se tendem a se envolver com as organizações. Os autores discutem, sob base da psicologia social e da economia comportamental, que os indivíduos, de acordo com sua orientação para valores sociais, podem ser categorizados em dois tipos principais self-regarding e reciprocal.

Segundo Bridoux e Stoelhorst (2014), os indivíduos que se preocupam apenas com seus ganhos pessoais e não valorizam a justiça como um todo, são atores individualistas, que se enquadra na classificação de self-regarding, e stakeholders com esta característica, tende a se relacionar e se envolver com organizações com baixa orientação para stakeholders. Por outro lado, atores classificados como reciprocals, cuja característica é pró-social, tendem a recompensar tratamentos justos e punir tratamento injusto de si mesmos ou de outros, mesmo 
que afete a própria recompensa. Neste tipo de stakeholder, cuja característica volta-se para um relacionamento recíproco, espera-se que os stakeholders se envolvam com organizações cuja orientação seja voltada a gestão para stakeholders.

A partir disso, propõe-se que, dependendo do tipo e das características dos stakeholders, a participação na tomada de decisão pode ser potencializada ou reduzida. Ou seja, o tipo de stakeholder modera a relação entre priorização e participação e entre reciprocidade e participação. Em função disso, sugere-se que:

Proposição 3: O tipo de stakeholder modera a relação entre a prioridade do stakeholder e a participação no processo de decisão organizacional.

Proposição 4: As características do stakeholder moderam a relação entre a reciprocidade no relacionamento com o stakeholder e a participação no processo de decisão organizacional.

\section{Considerações Finais}

O intenso desenvolvimento da teoria dos stakeholders nas três últimas décadas vem alavancando o emprego de seus conceitos e modelos em diversos campos da área de gestão, tais como governança corporativa, responsabilidade social corporativa, estratégia empresarial e ética empresarial. Todavia, apesar deste significativo desenvolvimento há diversos pontos a serem resolvidos. Pontos em que o estado da arte da teoria ainda não foi capaz de equacionar, a exemplo do pleno entendimento daquilo que cria valor aos stakeholders, como são estabelecidos os relacionamentos e a importância do envolvimento e participação dos stakeholders nos processos da organização, em especial na decisão organizacional, foco deste ensaio teórico.

O estado da arte na teoria dos stakeholders encontra-se em uma crescente linha de pesquisa que desenvolve uma explicação alternativa de como os gestores definem suas estratégias, criam e distribuem valor. Esta linha de pensamento defende que a justiça e a reciprocidade melhor explicam as decisões dos gestores. Tal pensamento assume que este tipo de tratamento obtém maior engajamento e consequente criação de valor para a empresa por parte dos stakeholders (Bosse et al., 2009; Harrison et al., 2010; Harrison e Bosse, 2013). Esta explicação considera uma positiva e negativa reciprocidade dos stakeholders em função da 
percepção destes quanto à justiça ou injustiça praticada pela empresa (Bosse et al., 2009). Em função desta perspectiva, nos últimos anos vários pesquisadores, entre eles Freeman (2010), Harrison et al. (2010), Garcia-Castro e Aguilera (2015), Tantalo e Priem (2016) e Jones, Harrison e Felps (2018), voltaram sua atenção para a perspectiva de criação de valor e para os processos organizacionais que são atrelados a este objetivo.

Este ensaio teórico contribui para o entendimento do fenômeno da gestão para stakeholderse a participação no processo de decisão organizacional, o qual, até o momento não encontrou estudos empíricos que demonstrem evidências de como os gestores ponderam o tratamento aos stakeholders em função da sua participação, envolvimento e capacidade de criação de valor no processo decisório. Neste sentido, esta proposta é original, tocando no estado da arte desta literatura.

Embora o trabalho discuta o tema ainda de forma embrionária, pode-se verificar um potencial de investigação de questões que o permeiam o tema. Uma destas questões refere-se a quais os limites para a participação dos stakeholders na tomada de decisão para que haja benefício mútuo ao invés de aumento de conflitos de interesses ou de alianças políticas. Outro ponto é como operacionalizar empiricamente e operar gerencialmente um relacionamento de reciprocidade entre a organização e o stakeholder. Além disto, questiona-se até que ponto a organização está disposta a compartilhar informações e, em troca disso, os stakeholders tenham acesso as informações da organização.

As proposições levantadas neste estudo buscam avançar em direção ao objetivo de aprimorar e fomentar pesquisas futuras sobre o relacionamento com stakeholders nos processos de decisão organizacional. Neste sentido, estudos futuros podem aprofundar a discussão, bem como operacionalizar empiricamente as proposições levantadas sob o contexto da abordagem da teoria de stakeholders.

\section{Referências}

Abrahamson, E., \& Baumard, P. (2008). What lies behind organizational façades and how organizational façades lie: An untold story of organizational decision making. The Oxford handbook of organizational decision making, 437-452. 
Adams, R., Licht, A., \& Sagiv, L. (2011). Shareholders and stakeholders: How do directors decide? Strategic Management Journal, 32(12), 1331-1355.

Anbarasan, P. (2018). Stakeholder Engagement in Sustainable Enterprise: Evolving a Conceptual Framework, and a Case Study of ITC. Business Strategy and the Environment, 27(3), 282-299.

Ashmos, D. P., Duchon, D., \& McDaniel, R. R. (1998). Participation in strategic decision making: The role of organizational predisposition and issue interpretation. Decision Sciences, 29(1), 25-51.

Barbero, E., \& Marchiano, M. (2016). Stakeholders or Shareholders? Board members' personal values and corporate identity. Revista Brasileira de Gestão de Negócios, 18(61), 348369.

Beaucamp, T. L., \& Bowie, N. E. (2001). Ethical Theory and Business. (6a ed.). Prentice Hall. 45-105.

Boaventura, J. M. G., Cardoso, F. R., Silva, E. S., \& Silva, R. S. (2009). Teoria dos Stakeholders e Teoria da Firma: um estudo sobre a hierarquização das funções-objetivo em empresas brasileiras. Revista Brasileira de Gestão de Negócios, 11(32), 289-307.

Bosse, D. A., Phillips, R. A., \& Harrison, J. S. (2009). Stakeholders, Reciprocity, And Firm Performance. Strategic Management Journal, 30(4), 447-456.

Buysse, K., \& Verbeke, A. (2003). Proactive environmental strategies: a stakeholder management perspective. Strategic Management Journal, 24(5), 453-470.

Bridoux, F., \& Stoelhorst, J. W. (2014). Microfoundations for stakeholder theory: Managing stakeholders with heterogeneous motives. Strategic Management Journal, 35(1), 107125.

Bridoux, F., \& Stoelhorst, J. W. (2016). Stakeholder relationships and social welfare: A behavioral theory of contributions to joint value creation. Academy of Management Review, 41(2), 229-251.

Cameron, B. G., Crawley, E. F., Feng, W., \& Lin, M. (2011). Strategic Decisions in Complex Stakeholder Environments: A Theory of Generalized Exchange. Engineering Management Journal, 23(3), 37-45. 
Chakhar, S., \& Saad, I. (2014). Incorporating stakeholders' knowledge in group decisionmaking. Journal of Decision Systems, 23(1), 113-126.

Chigona, W., Roode, D., Nabeel, N., \& Pinnock, B. (2010). Investigating the impact of stakeholder management on the implementation of a public access project: The case of Smart Cape. South African Journal of Business Management, 41(2), 39-49.

Child, J., Elbanna, S. A. I. D., \& Rodrigues, S. (2010). The political aspects of strategic decision making. The handbook of decision making, 105-137.

Clarkson, M. B. E. (1995). A Stakeholder Framework for Analyzing and Evaluating Corporate Social Performance. Academy of Management Review, 20(1), 92-117.

Cobb, J. A. (2016). How firms shape income inequality: Stakeholder power, executive decision making, and the structuring of employment relationships. Academy of Management Review, 41(2), 324-348.

Delmas, M., \& Toffel, M. (2008). Organizational responses to environmental demands: Opening the black box. Strategic Management Journal, 29(10), 1027-1055.

Dye, K. C., Eggers, J. P., \& Shapira, Z. (2014). Trade-offs in a tempest: Stakeholder influence on hurricane evacuation decisions. Organization Science, 25(4), 1009-1025.

Edelenbos, J., \& Klijn, E. H. (2006). Managing stakeholder involvement in decision making: A comparative analysis of six interactive processes in the Netherlands. Journal of Public Administration Research and Theory: J-PART, 16, 417-446.

Evan, W. M., \& Freeman, R. E. (1993). A stakeholder theory of the modern corporation: Katian capitalism. In: Donaldson, T.; Werhane, P. H. (Eds.) Ethical issues in business. Englewood Cliffs: Prentice-Hall, 166-71.

Finkelstein, S., Hambrick, D. C., \& Cannella, A. A. (2009). Strategic leadership: Theory and research on executives, top management teams, and boards. Strategic Management (Oxford U.)

Freeman, R. E. (1984). Strategic management: a stakeholder approach. Boston: Pitman.

Freeman, R. E. (2010). Managing for Stakeholders: Trade-offs or Value Creation. Journal of Business Ethics, 96(S1), 7-9. 
Freeman, R. E., Harrison, J. S., \& Wicks, A. C. (2007). Managing for Stakeholders: Survival, Reputation, and Success. New Haven: Yale University Press.

Freeman, R. E., Phillips, R., \& Sisodia, R. (2018). Tensions in Stakeholder Theory. Business \& Society.

Friedman, A. L. \& Miles, S. (2006). Stakeholders: theory and practice. New York: Oxford University Press.

Frooman, J. (2010). The issue network: Reshaping the stakeholder model. Canadian Journal of Administrative Sciences/Revue Canadienne des Sciences de l'Administration, 27(2), 161-173.

Garcia-Castro, R., \& Aguilera, R. V. (2015). Incremental value creation and appropriation in a world with multiple stakeholders. Strategic Management Journal, 36(1), 137-147.

Greenwood, M. (2007). Stakeholder engagement: Beyond the myth of corporate responsibility. Journal of Business Ethics, 74(4), 315-327.

Harrison, J. S., \& Bosse, D. A. (2013). How much is too much? The limits to generous treatment of stakeholders. Business Horizons, 56(3), 313-322.

Harrison, J. S., Bosse, D. A., \& Phillips, R. A. (2010). Managing for stakeholders, stakeholder utility functions, and competitive advantage. Strategic Management Journal, 31(1), 5874.

Henriques, I., \& Sadorsky, P. (1999). The relationship between environmental commitment and managerial perceptions of stakeholder importance. Academy of Management Journal, 42(1), 87-99.

Heugens, P. P., Van Den Bosch, F. A., \& Van Riel, C. B. (2002). Stakeholder integration: Building mutually enforcing relationships. Business \& Society, 41(1), 36-60.

Huijstee, M., \& Glasbergen, P. (2008). The practice of stakeholder dialogue between multinationals and NGOs. Corporate Social Responsibility and Environmental Management, 15(5), 298-310.

Jensen, M. C. (2001). Value Maximisation, Stakeholder Theory, and the Corporate Objective Function. European Financial Management, 7(3), 297-317. 
Johnson-Cramer, M. E. (2004). Organization-Level Antecedents of Stakeholder Conflict. In Academy of Management Proceedings, vol. 2004: F1-F6. Academy of Management.

Jones, T. M., Harrison, J. S., \& Felps, W. (2018). How applying instrumental stakeholder theory can provide sustainable competitive advantage. Academy of Management Review, 43(3), 01-53.

Jones, T. M., Wicks, A. C., \& Freeman, R. E. (2017). Stakeholder theory: The state of the art. The Blackwell guide to business ethics, 17-37.

Kleine, M., Langenbach, P., \& Zhurakhovska, L. (2016). Fairness and persuasion: How stakeholder communication affects impartial decision making. Economics Letters, 141, 173-176.

Krick, T., Forstater, M., Monaghan, P., \& Sillanpää, M. (2005). The stakeholder engagement Manual Volume 2: The practitioners' handbook on stakeholder engagement. Account Ability, the United Nations Environment Programme, and Stakeholder Research Associates.

MacDonald, A., Clarke, A., \& Huang, L. (2018). Multi-stakeholder Partnerships for Sustainability: Designing Decision-Making Processes for Partnership Capacity. Journal of Business Ethics, 1-18.

Mascena, K. M. C., Kim, J., Fischmann, A. A., \& Corrêa, H. L. (2015). Priorização de Stakeholders: Contribuição dos estudos teóricos e empíricos. Revista de Administração da UFSM, 8, 42-59

Mitchell, R. K., Agle, B. R., \& Wood, D. J. (1997). Toward a Theory of Stakeholder Identification and Salience: Defining the Principle of Who and What Really Counts. Academy of Management Review, 22(4), 853-886.

Morsing, M., \& Schultz, M. (2006). Corporate social responsibility communication: stakeholder information, response and involvement strategies. Business Ethics: A European Review, 15(4), 323-338.

Noland, J., \& Phillips, R. (2010). Stakeholder engagement, discourse ethics and strategic management. International Journal of Management Reviews, 12(1), 39-49. 
Nystrom, P. C., \& Starbuck, W. H. (1984). Managing beliefs in organizations. The Journal of Applied Behavioral Science, 20(3), 277-287.

O’Brien, J., \& David, P. (2014). Reciprocity and R\&D search: Applying the behavioral theory of the firm to a communitarian context. Strategic Management Journal, 35(4), 550-565.

Parmar, B. L., Freeman, R. E., Harrison, J. S., Wicks, A. C., Purnell, L., \& De Colle, S. (2010). Stakeholder theory: The state of the art. The academy of management annals, 4(1), 403445.

Pedersen, E. R. G. (2011). All animals are equal, but...: management perceptions of stakeholder relationships and societal responsibilities in multinational corporations. Business Ethics: A European Review, 20(2), 177-191.

Phillips, R. (2003a). Stakeholder theory and organizational ethics. Berrett-Koehler Publishers: San Francisco.

Phillips, R. (2003b). Stakeholder legitimacy. Business Ethics Quarterly, 13(1), 25-41.

Phillips, R., Freeman, R. E., \& Wicks, A. C. (2003). What stakeholder theory is not. Business ethics quarterly, 13(4), 479-502.

Rasche, A., \& Esser, D. E. (2006). From stakeholder management to stakeholder accountability. Journal of Business Ethics, 65(3), 251-267.

Rühli, E., Sachs, S., Schmitt, R., \& Schneider, T. (2017). Innovation in multistakeholder settings: The case of a wicked issue in health care. Journal of Business Ethics, 143(2), 289-305.

Saravanamuthu, K. (2018). How risk information and stakeholder-participation affect the sustainability of collaborative decisions: A case study on how the sustainability of stakeholder decisions is affected by different levels of stakeholder participation in preparing risk information. Business Strategy and the Environment, 1-12.

Simons, L. P., \& Wiegel, V. (2009). Evaluating AHP as multi-stakeholder decision tool. In Technology Management Conference (ICE), 2009 IEEE International (pp. 1-9).

Stevens, J. M., Steensma, H. K., Harrison, D. A., \& Cochran, P. L. (2005). Symbolic or substantive document? The influence of ethics codes on financial executives' decisions. Strategic Management Journal, 26(2), 181-195. 
Sundaram, A. K., \& Inkpen, A. C. (2004). The Corporate Objective Revisited. Organization Science, 15(3), 350-363.

Schwartz, S. H. (1992). Universals in the content and structure of values: Theoretical advances and empirical tests in 20 countries. In Advances in experimental social psychology (Vol. 25, pp. 1-65). Academic Press.

Tantalo, C., \& Priem, R. L. (2016). Value creation through stakeholder synergy. Strategic Management Journal, 37(2), 314-329.

Vazquez-Brust, D. A., Liston-Heyes, C., Plaza-Úbeda, J. A., \& Burgos-Jiménez, J. (2010). Stakeholders pressures and strategic prioritisation: An empirical analysis of environmental responses in Argentinean firms. Journal of Business Ethics, 91(2), 171192.

Yu, E. P. Y., Guo, C. Q., \& Luu, B. V. (2018). Environmental, social and governance transparency and firm value. Business Strategy and the Environment, 1-18. 